\title{
Correction to: Cobalt(II)/(III)-Lanthanide(III) Complexes as Molecular Magnets
}

\author{
Atanu Dey, Shalini Tripathi, Maheswaran Shanmugam, \\ Ramakirushnan Suriya Narayanan, and Vadapalli Chandrasekhar
}

\section{Correction to:}

Chapter "Cobalt(II)/(III)-Lanthanide(III) Complexes

as Molecular Magnets" in: A. Dey et al.,

Topics in Organometallic Chemistry,

DOI: $10.1007 / 3418 \_2018 \_9$

In the Chapter opener page, the spelling of the author is inadvertently misspelt as Vadapalli Chandrsekhar, which is now corrected as Vadapalli Chandrasekhar. 